Martin Weißmann*

\title{
Niklas Luhmann als Kritiker des Postulats der Werturteilsfreiheit. Zum soziologisch disziplinierten Möglichkeitssinn systemtheoretischer Kritik
}

DOI 10.1515/sosys-2015-0015

Zusammenfassung: Insbesondere in seinen frühen Arbeiten kritisiert Niklas Luhmann das Postulat einer werturteilsfreien Soziologie und präsentiert seine Systemtheorie als deskriptiv-erklärende und normativ-bewertende Theorie der sozialen Welt. Der Artikel rekonstruiert diesen bislang kaum bekannten Aspekt in Luhmanns Werk und vertritt die These, dass die Methode des Äquivalenzfunktionalismus und das Verständnis sozialer Systeme als wertkomplexer Problemlösungszusammenhänge die Grundlage für ein Konzept systemtheoretischer Sozialkritik bilden, welches eine Alternative zu den Ansätzen immanenter Kritik einerseits und werturteilsfreier Sozialforschung andererseits bietet. Wichtige Grenzen systemtheoretischer Kritik liegen darin, dass ihre wertenden Aussagen stets auf konkrete Systemreferenzen und Bezugsprobleme bezogen sein müssen; dass die Selektion dieser Bezugsprobleme auch des Rekurses auf wissenschaftlich nicht begründbare Werte bedarf und schließlich darin, dass ihre wertenden Urteile aus theorieimmanenten Gründen in der Regel nicht durch Eindeutigkeit auffallen, sondern von Ambivalenz geprägt sind.

Abstract: Especially in his early work Niklas Luhmann criticizes the idea of sociology as a value free discipline and demands to develop systems theory as a descriptive-explanatory and normative-evaluating theory of the social world. This article reconstructs this mostly ignored aspect of Luhmann's work and shows that the method of equivalence-functionalism combined with an understanding of social systems as a set of interdependent and often contradictory problems and their contingent solutions can serve as a foundation for a criticism based on systems theory as an alternative to the approach of immanent critique on the one hand and value-free social research on the other hand. Finally, the article considers important limitations of a criticism based on systems theory by concluding that its evaluative statements must always refer to a specific system of reference and specific functional problems. The selection of these points of reference inter alia

*Korrespondenzautor: Martin Weißmann, Fakultät für Soziologie, Universität Bielefeld, Postfach 100 131, D-33501 Bielefeld, email: martin.weissmann@uni-bielefeld.de 
depends on non-scientific values and the value judgments of a criticism based on systems theory are usually characterized by ambivalence instead of clarity and certainty.

\section{Die Bewertbarkeit des Sozialen als Reflexions- thema der Soziologie}

Die Rückkehr der Kritik in die Soziologie wird seit einigen Jahren mit gutem Recht behauptet und vorangetrieben. ${ }^{1}$ Die in diesem Heft versammelten Beiträge sind nicht das erste Zeugnis davon, dass das Thema mit einiger Verspätung nunmehr auch die systemtheoretisch denkenden Vertreter des Fachs erreicht hat (s. etwa Amstutz/Fischer-Lescano 2013; Kieserling 2014; Scherr 2015; Möller/Siri 2016). ${ }^{2}$ Als Anachronismus, als erneute Verhandlung längst gelöster und damit obsolet gewordener Probleme, wäre diese Rückkehr der Kritik falsch verstanden, da es sich bei dem Verhältnis von Soziologie und Kritik um eines der zentralen Reflexionsthemen des Fachs handelt (Kieserling 2004a, 16; Boltanski 2010, 35, 46). Jeder umfassende Versuch der Selbstbestimmung der Soziologie muss auch eine Antwort auf die Frage nach den Möglichkeitsbedingungen soziologischer Wertungen beinhalten. An dieser Frage wird verhandelt, wie die Soziologie sich vernünftigerweise gegenüber den Selbstbeschreibungen und Strukturen anderer sozialer Systeme verhalten kann und von welchen dieser Möglichkeiten sie Gebrauch machen soll. Eine erste Gruppe von Antworten der Fachgeschichte auf diese Frage hält eine Soziologie für vollständig, die registrierend zur Kenntnis nimmt und zu erklären versucht, was in der Sozialwelt der Fall ist. Eine zweite Gruppe meint dagegen, dass eine vollständige Wissenschaft des Sozialen mehr kann und mehr soll als dies, nämlich das, was der Fall ist, wertend in Hinblick auf seine Rationalität zu befragen.

Die Systemtheorie Niklas Luhmanns wird üblicherweise der ersten Gruppe von Antworten zugerechnet. Die bis heute mehrheitsfähige Lesart der System-

1 Die erneute Prominenz des Themas in der Doppelgestalt verschiedener kritischer Soziologien einerseits und Ansätzen zu einer Soziologie der Kritik andererseits ist auch in der deutschsprachigen Soziologie und Sozialphilosophie nicht zu übersehen. Siehe für die Soziologie etwa Dörre et al. 2009; Boltanski 2010; Vobruba 2013; Bröckling 2013; Lessenich 2014 und für die Sozialphilosophie Honneth 2007; Jaeggi/Wesche 2009; Stahl 2013; Jaeggi 2014.

2 Vereinzelt wurde die Thematik freilich auch in Bezug auf die Systemtheorie schon zuvor aufgegriffen, s. insbesondere Kneer (1996, 389-402) und des Weiteren etwa Japp 1991; Müller-Doohm 2001; Schroer 2004 sowie die in der folgenden Anmerkung genannte Literatur. 
theorie hält diese für eine notwendigerweise werturteilsfreie Soziologie, die sich für eine „normative Perspektive welcher Art auch immer“ nicht eigne (Wagner 2012, 429), da sie „keine wertenden Urteile“ über ihre Gegenstände fällen könne (Amstutz 2013, 386). ${ }^{3}$ Dieser Lesart widerspricht mein Beitrag ausgehend von einer Rekonstruktion der meines Wissens nach bislang nicht rezipierten Kritik am Postulat der Werturteilsfreiheit durch Niklas Luhmann. Dabei zeige ich, dass und aus welchen Gründen Luhmann die insbesondere mit dem Namen Max Webers (1904) verbundene Formel der Werturteilsfreiheit als Selbstbeschreibung und programmatische Grundlage seiner Theorie abgelehnt hat.

Im Gegensatz zu den Vertretern der ,Kritischen Systemtheorie“ (Amstutz/ Fischer-Lescano 2013) gehe ich davon aus, dass mit dem Konzept der immanenten Kritik auch die zweite wichtige Antwort der Fachgeschichte auf das skizzierte Reflexionsthema der Soziologie keine Grundlage für ein angemessenes Selbstverständnis der Systemtheorie bietet. Für immanente Kritiker von Hegel über Marx, Lukács und Adorno bis zu zeitgenössischen Vertreterinnen der Kritischen Theorie wie Rahel Jaeggi, Axel Honneth und Titus Stahl ist die Annahme konstitutiv, dass systematische Widersprüche in sozialen Gebilden in erste Linie einen diese Gebilde destabilisierenden Faktor darstellen und deshalb als eine „Form sozialer Irrationalität“ zu bezeichnen sind (Peters 1991, 148; vgl. Honneth 2014, 59; Jaeggi 2014, 383; Stahl 2013, 417). Diese Annahme kann von einer systemtheoretisch informierten Sozialkritik nicht geteilt werden. $\mathrm{Zu}$ den zentralen Einsichten der Systemtheorie und anderer soziologischer Theorien zählt ja gerade, in dem „Einbau von internen Unbestimmtheiten, Freiheiten und Widersprüchen in das System“ (1967b, 79) ${ }^{4}$ eine funktional sinnvolle und oft unverzichtbare Reaktion auf die Ungeordnetheit, Differenziertheit und Widersprüchlichkeit von Systemumwelten zu sehen. Der Systemtheorie (aber nicht nur ihr) gilt das Maß des einem sozialen Gebilde bekömmlichen Selbstwiderspruchs - etwa zwischen seinen manifesten und seinen latenten Strukturen oder zwischen den ihm zugehörigen Teilsystemen - deshalb im Gegensatz zur immanenten Kritik als eine Variable, die positiv mit der Komplexität der jeweiligen Umwelt des Systems korreliert. ${ }^{5}$

3 Ähnlich urteilen auch Pollack 1991, 97; Füllsack 1997, 321; Wagner 2005, 48; Gebhard et al. 2006, 279; Horster 2008, 376, 389; Pahl 2009, 255; Richter 2011, 271 f.; Stäheli 2012, 40 f.; Wagner 2013, 79.

4 Hier und im Folgenden handelt es sich bei Literaturangaben ohne Autorenangabe um Verweise auf Texte Niklas Luhmanns.

5 Diese Einschätzung widerspricht dem Urteil vieler Vertreter der ,Kritischen Systemtheorie“, die der Systemtheorie nicht nur den Begriff der „,immanenten Kritik“ empfehlen, sondern auch die für diese Kritikform konstitutive Annahme bejahen, widersprüchliche Zustände seien wesentlich dysfunktional und instabil (vgl. etwa Fischer-Lescano 2013, 15 f.; Amstutz 2013, 389). 
Im zweiten Teil dieses Textes charakterisiere ich daher eine Form systemtheoretischer Kritik, die der Selbstbeschreibung der Systemtheorie eine Alternative zu den Formeln der Werturteilsfreiheit und der immanenten Kritik anbietet. Die These ist, dass systemtheoretische Kritik als disziplinierte Bewertung der Strukturen, Selbstvereinfachungen und Selbstverständlichkeiten der jeweils kritisierten Systeme begriffen werden kann. Diszipliniert ist diese Form der Kritik einerseits durch die theoretischen und empirischen Einsichten ihrer Disziplin, andererseits durch die wirklichen Möglichkeitsspielräume der von ihr analysierten und kritisierten sozialen Systeme, in deren Erkundung die wichtigste Aufgabe der Kritik liegt. $\mathrm{Zu}$ den Möglichkeiten systemtheoretischer Kritik zählt es dann auch, realisierte und mögliche Problemlösungen äquivalenzfunktionalistisch vergleichend in Bezug auf ihre Brauchbarkeit für wertkomplexe Sozialsysteme zu bewerten. ${ }^{6}$

\section{Luhmanns Kritik am Postulat der Werturteilsfreiheit}

Luhmann hat seiner Theorie zuweilen die Aufgabe zugewiesen, „kritisch auch in Bezug auf den kritischen Gestus der kritischen Theorie“ zu sein (1993d, 54). Wie oben belegt, wird üblicherweise angenommen, dass diese Kritik der Kritiker von Luhmann auf Grundlage einer sich in Bezug auf die Gegenstände der Soziologie werturteilsfrei verstehenden Theorie formuliert worden ist. Gegen diese Lesart zeige ich, dass Luhmann nicht nach einer werturteilsfreien Systemtheorie, sondern nach einer spezifischen Form systemtheoretischer Wertungen

Diese Annahme scheint mir schlecht zu einer an Luhmann orientierten Systemtheorie zu passen, der zufolge ein System nichts anderes als ein geordneter Widerspruch ist (vgl. etwa 1964b, 74 f., 239 f.). Siehe dazu auch Kieserling (2014; 2015).

6 Ich verstehe ,Kritik‘ in Übereinstimmung mit den Vertretern der Kritischen Theorie (vgl. etwa Jaeggi 2009, 282f.) ebenso wie mit denen einer pragmatischen Soziologie der Kritik (vgl. etwa Boltanski 2010, 26) und dem seit dem 18. Jahrhundert insgesamt vorherrschenden Begriffsverständnis (vgl. Röttgers 1982) als ein „Urteil über den Wert einer Sache“ (Bittner 2009, 136), also als ein eindeutig normatives Unternehmen. Kritik als Anwendung einer bewertenden Unterscheidung ist dabei nicht exklusiv auf Ablehnung oder Bejahung und auch nicht exklusiv auf eine einzige Systemreferenz wie etwa die moderne Gesellschaft festgelegt. Die Rechtfertigung dieser Begriffsfassung liegt in der These, dass sie gut zu dem skizzierten Reflexionsproblem der Soziologie passt, für dessen Bearbeitung es keinen Unterschied macht, ob die Möglichkeit soziologischen Lobs oder soziologischen Tadels in Frage steht und welches die davon betroffenen Gegenstände sind, da es zunächst um die Möglichkeit gegenstandsbezogener soziologischer Wertungen überhaupt geht. 
gesucht hat. Einen Beleg für diese These bietet Luhmanns kaum bekannte Kritik am Postulat der Werturteilsfreiheit, die am deutlichsten in den frühen Arbeiten des Autors zum Ausdruck kommt.

In zahlreichen Texten aus den 1960er Jahren, die sich insbesondere mit Themen an den Schnittstellen von Verwaltungswissenschaft, Rechtswissenschaft und (Organisations-)Soziologie beschäftigen, diagnostiziert Luhmann eine Zweiteilung der Wissenschaften vom menschlichen Handeln: Sie ließen sich entweder der empirisch-deskriptiv-erklärenden oder aber der normativpräskriptiven, auf eine Rationalisierung des Handelns abzielenden Forschung zuordnen. Die Trennung dieser beiden Formen der Wissenschaft führt Luhmann auf das 19. Jahrhundert zurück, mit dessen Ausgang die Annahme einer Einheit von existierender und vernünftiger Ordnung nachhaltig an Überzeugungskraft verloren habe (1966a, 22f.). Seitdem würden die Fragen nach dem wirklichen und dem vernünftigen Handeln zunehmend getrennt voneinander mit unterschiedlichen Begriffen und anschließend an unterschiedliche Denktraditionen bearbeitet: Normative Wissenschaften ,wollen den Handelnden über sein richtiges Handeln aufklären“, während empirische Wissenschaften sich als wertfrei verstehen und sich darum bemühen, „faktische Handlungsverläufe zu erklären oder gar vorauszusagen“ (1966a, 23). Die Soziologie seiner Zeit ordnet Luhmann dieser deskriptiv-wertfreien Wissenschaft zu, die „den Wissenschaften vom rationalen Wirtschaften und rationalen Organisieren“ mit einer „gewissen Scheu“ begegnet und Wert darauf legt, „sich eines eigenen kritischen Urteils über die Richtigkeit des Handelns“ zu enthalten (1965b, 7 f.). Zu den normativen Wissenschaften zählt Luhmann dagegen neben der Ethik auch die klassische Organisations-, Betriebswirtschafts- und Rechtslehre sowie große Teile der Politikwissenschaft (o.J., 205; 1964a, 46).

Das Verhältnis dieser zwei Formen der Forschung zueinander ist für den frühen Luhmann ein außergewöhnlich wichtiges Thema. Er greift es in fast jeder Schrift dieser Schaffensperiode an zumeist zentraler Stelle auf und betont, dass es sich nicht um ein methodologisches Spezialthema, sondern um eine Frage von grundlegender Bedeutung für jede Sozialwissenschaft handelt (1968b, 343). Den heutigen Leser mag es überraschen, dass Luhmann an dem diagnostizierten „Schisma rational-normierenden und empirisch-erklärender Handlungswissenschaften“ (1966a, 26) starke Kritik äußert und mit dem Anspruch auftritt, „den Trenngraben zuzuschaufeln“ (1965b, 19), „diese Kluft von Soziologie und normativem Rationalismus zu überbrücken“ (o.J., 205). Der Autor wird nicht müde, die „Notwendigkeit einer Synthese“ deskriptiver und normativer Perspektiven zu betonen (1968b, 312), die es erlauben würde, „die Einseitigkeit von nur-normativen bzw. nur-empirischen Methoden zu vermeiden“ (1966a, 116). Er nennt die Isolierung präskriptiver und deskriptiver Forschungsansätze eine „Krise“ und 
bedauert die Soziologie dafür, „zu früh“ in sie „hineingeboren“ zu sein (1970a, 141). Kurz: „empirisches und normatives Forschen verbinden“ $(1966 a, 116) \mathrm{zu}$ wollen, ist ein wichtiges und heute kaum bekanntes Forschungsprogramm des frühen Luhmann.

Diese Programmatik findet ihren Ausdruck auch in einer Kritik des Postulates der Werturteilsfreiheit. Luhmann betont, dass die „unselige Formel der Wertfreiheit“ (1965b, 202) einer äußerst oberflächlich geführten Diskussion entspringe (vgl. 1969, 254). Als „Symbol vergangener Neurosen“ gehöre sie allenfalls noch „in akademische Festreden“ (1969, 263), könne aber nicht beanspruchen, zeitgemäße Forschungen anzuleiten. Die Betonung der Selbstverständlichkeit, dass persönliche Wertpräferenzen des Forschers „für die Wahrheit seiner Theorie ... ebenso irrelevant wie Tabletten und Bücher“ sind (wenngleich all dies für die kausale Entstehung einer Theorie bedeutsam gewesen sein mag), lasse die entscheidende Frage „ob und wieweit eine Theorie sich $\mathrm{zu}$ den Wertungen ihres Gegenstandsfeldes kritisch einstellen kann“, gänzlich unbeantwortet (1969, 255, meine Hervorhebung, MW). Luhmann sucht nach einer Soziologie, die zwar auf Sozialtechnokratie, nicht aber zugleich auf „Wertungen schlechthin“ verzichtet (1967b, 68). Freilich weiß Luhmann auch um die Brisanz und Angreifbarkeit seiner programmatischen Thesen: „Jeder Schritt in Richtung auf Empfehlungen für richtiges Handeln würde wohlgerüstete Gegner auf den Plan rufen, die ihre Waffen aus den Arsenalen des Wertfreiheitsstreites beziehen. Allein die Erinnerung an diese schreckliche Zeit, und nicht etwa entschiedene Fragen, behindern uns heute.“( (o.J., 205)

Dass Luhmanns Programmatik als schlichte Verschmelzung deskriptiver und normativer Forschung missverstanden wäre, zeigen etwa seine Äußerungen zu Entscheidungstheorien, in denen er die moderne Form normativer Forschung ausgemacht hat. Nichts liegt ihm an einer Vereinheitlichung von Entscheidungstheorie und Systemtheorie (1968b, 345), viel jedoch an dem Versuch, ihre Differenzierung so zu gestalten, dass sie ,eine gemeinsame Aufklärungsarbeit ermöglicht und die gemeinsame Leistung steigert“ (1967b, 81; vgl. 1964b, 405; 1965b, 203). In einem ersten Schritt betont Luhmann die Differenz der Forschungsperspektiven, die er vor allem darin sieht, dass die normativen Wissenschaften in der Regel an die Perspektive der Handelnden gebunden bleiben. Sie befassen sich „stets mit der Ausarbeitung, Begründung oder Korrektur des gemeinten Sinnes einer Handlung, setzen also im Erlebnisraum des Handelnden an“ (1964a, 46). Die „Orientierungsvorstellungen des täglichen Lebens“ (o.J., 206) wie Zweck und Mittel sind zugleich die Grundbegriffe dieser Wissenschaften, die sich oft sogar darauf beschränken, „das Wunschdenken des Regelsetzers nachzuzeichnen“ (1964b, 308). Rationalisierung des Sozialen meint aus Sicht dieser Wissenschaften daher „immanente Kritik im Horizont des Handelns“ (o.J., 206). Eine soziologi- 
sche Theorie und Kritik des Sozialen muss Luhmann zufolge diese Beschränkungen hinter sich lassen, „den Horizont des Handelnden transzendieren“ (1965a, 312 f.; vgl. 1968b, 343), etwa auch latente Funktionen thematisieren (1966a, 24) und soziales Handeln nicht als isolierten Sachverhalt, sondern als Element sozialer Systeme zu begreifen versuchen. Systemtheoretische Kritik nach Luhmann traut sich zu, Distanz zu den Prämissen und dem Erleben der Handelnden einzunehmen, weil sie darum weiß, dass „nicht nur Annährung an den Gegenstand, sondern auch Distanznahme zum Erkennen“ führt (1967b, 68). Im Unterschied zu vielen anderen Formen der Sozialkritik will sie darauf verzichten, „die Wertprämissen der Entscheidenden unbesehen als Prämissen der Theorie zu übernehmen“ (1968b, 117).

Luhmanns Forschungsprogramm stellt die Differenz normativer und deskriptiver Aussagen und der sie hervorbringenden Wissenschaften also in Rechnung, tritt davon ausgehend aber mit dem überraschenden Anspruch auf, dieser Trennung vorgelagert zu sein und deshalb „nicht ausschließlich auf empirische bzw. normative Aussageform festgelegt werden“ zu können (1968b, 349). Luhmann konzipiert seine Soziologie mithin als Einheit weiterhin voneinander unterscheidbarer deskriptiver und normativer Forschung, als Analyse und Kritik des Sozialen. Die ihr „eigentümliche Art von Rationalität“ gewinnt diese Soziologie durch eine sowohl „anspruchsvolle“ als auch „methodisch bewußte Ausweitung des Handlungshorizontes“ in Form eines „nicht an Handlungszwecke gebundenen, abstrahierenden Vergleichs mit anderen Handlungsmöglichkeiten“: „Sie konfrontiert unter den Problemgesichtspunkten der Systemerhaltung, die nicht die Werte des Handelnden zu sein brauchen, alles Handeln mit anderen Möglichkeiten.“ (1965b, 202f.) Alles Handeln problematisieren zu wollen heißt auch, dass systemtheoretische Kritik den ihr zugänglichen Bereich im Vergleich vor allem zu der konditionalen Kritik Max Webers (1917, 44; 1919, 37 f.; vgl. Dahms 2013, 79) und anderen Formen interner Kritik etwa bei Gunnar Myrdal (1944; 1971, 109-113) immens erweitert. Nicht nur die Wahl von Mitteln, sondern auch die Setzung von Zwecken und sogar das Prinzip der Zwecksetzung überhaupt erscheint ihr als eine Variable, die dem kritischen (bewertenden) Vergleich mit anderen Möglichkeiten ausgesetzt werden kann.

Angesichts der großen Präsenz der Suche nach einer zu eigenständigen Wertungen fähigen Theorie im Frühwerk Luhmanns liegt die Frage nahe, ob eine solche Perspektive auch zu der späteren Systemtheorie Luhmanns passt, die stärker auf die Fremdbeschreibung sozialer Systeme ausgerichtet ist (vgl. Schmidt 2013, 331 ff. für eine verneinende Antwort in Bezug auf die rechtstheoretischen Schriften Luhmanns). Nach meinem Eindruck ließe sich in ausführlicheren Textanalysen zeigen, dass die Arbeit an einer wertfähigen, insofern kritischen 
und zugleich in Bezug auf die Praxis (auch anderer Disziplinen) ${ }^{7}$ kontaktfähigen Theorie ein wichtiges Merkmal des Gesamtwerkes Luhmanns ist, also keineswegs im Laufe der 1970er oder 1980er Jahre abbricht. Hingewiesen sei diesbezüglich neben den Belegen im folgenden Abschnitt nur auf Luhmanns Abschiedsvorlesung aus dem Jahr 1993. An dieser prominenten Stelle problematisiert Luhmann die verloren gegangene Einheit der Soziologie (1993e, 246), die es erschwere, normative und empirische Forschung in einen Zusammenhang zu bringen (245, 252), moniert einen Mangel an interdisziplinärer Kontaktfähigkeit des Fachs (251) und schließlich auch das Vergessen der Frage nach dem Zusammenhang von Theorie und Praxis (249). Luhmann arbeitet heraus, dass die Soziologie eine an die Frage, ,Was ist der Fall?‘ gebundene positivistische sowie eine an die Frage ,Was steckt dahinter?' gebundene kritische Tradition ausgebildet hat $(245,257)$ und würdigt die Bedeutsamkeit beider Traditionen mit dem Hinweis, dass kein wichtiger Soziologe die bei ihm jeweils weniger prominente Ausrichtung gänzlich ausblenden konnte, da die „Spannung“ zwischen ihnen auf ein „Theorieproblem“ verweist, „das die Soziologie seit ihrem Beginn geprägt hat“ (245). Und schließlich stellt Luhmann erneut die Frage, die ihn schon in seinen Schriften der 1960er und 1970er Jahre beschäftigte: Warum es „auf immer unmöglich bleiben“ sollte, die Fragestellungen der deskriptiv-positivistischen Soziologie mit denjenigen der normativ-kritischen Soziologie zusammenzuführen (252). Zu dieser Programmatik passt auch die vom ,späten‘ Luhmann geäußerte Einschätzung, die Autoren der Frankfurter Schule würden zu wenig Kritik formulieren, da sie durch ihre Fokussierung auf die Semantik von Kapital und Arbeit zu wenige Aspekte der modernen Gesellschaft problematisieren (1988, 171; 1990, 648).

\section{Systemtheoretische Kritik als Suche nach „weniger schmerzlichen Zuständen“}

Luhmanns weitreichender Anspruch, normative und deskriptive Soziologie miteinander zu verbinden, ohne sie zu vereinheitlichen, kann nur von einer Theorie umgesetzt werden, die über einen für beide Ausrichtungen der Soziologie verwendbaren begrifflichen Bezugsrahmen verfügt (vgl. 1966a, 24). Wiederum gibt Luhmann selbst hilfreiche Hinweise auf deskriptiv und normativ nutzbare Konzepte der Systemtheorie:

7 Zur konstant bleibenden Wertschätzung und Einforderung interdisziplinär orientierter Theoriebildung im Spätwerk Luhmanns siehe etwa 1983, 62; 1990, 240, 680. 
Systemkonzeption und Methode tragen zusammen dazu bei, die Kluft zwischen empirischfeststellender und rational-normierender Betrachtungsweise zu überbrücken. Der skizzierte Forschungsansatz läßt sich keiner dieser Perspektiven einordnen. Er ist ihrer Trennung vorgelagert und läßt sich für beide Arten von Zielsetzungen verwenden: zur Untersuchung der empirischen Frage, welche Handlungsmöglichkeiten bestimmte Funktionen mit welchen Folgeproblemen erfüllen oder erfüllen können; und zur Beantwortung der Frage, welche Handlungen in bestimmten konkreten Systemen systemrational sind, das heißt: als Beitrag zur Lösung der Probleme dieses Systems empfohlen werden können. (1966a, 111 f.) ${ }^{8}$

Das angeführte Zitat benennt wesentliche Aspekte systemtheoretischer Kritik, deren nun folgende Explikation erste Hinweise auf die Methodologie systemtheoretischen Wertens geben soll. Es geht mir also um die Frage, wie die Systemtheorie wertet. Was sind die Grundlagen, Methoden und Ziele ihrer Kritik der Sozialwelt? Wie und wann kann es ihr gelingen, Soziales „als System kritisch“ zu betrachten? (1966a, 116)

Ein wichtiger Ausgangspunkt aller Antworten auf diese Fragen ist, dass die wertenden Aussagen der Systemtheorie an konkrete Systeme gebunden sein müssen. Adorno benennt den Grund für diese Begrenzung der Kritik mit dem Hinweis, dass die soziale Welt „nicht in sich rational kontinuierlich“ (Adorno 1972, 9) ist. In einer fast identischen Formulierung weist Luhmann darauf hin, dass in der modernen Gesellschaft kein systemübergreifendes „Rationalitätskontinuum“ existiert (o.J. [1982], 192, 201). Mit der Kritischen Theorie ist sich die Systemtheorie mithin darin einig, dass die in verschiedenen sozialen und psychischen Systemen vorherrschenden Rationalitätsvorstellungen, also Vorstellungen darüber, was (zu tun) richtig und wichtig ist, aus sozialstrukturellen Gründen weder identisch noch notwendigerweise aufeinander abgestimmt sind. Jede

8 Luhmann will also die Forderung der Werturteilsfreiheit umgehen, ohne gegen Humes Diktum der wechselseitigen logischen Unableitbarkeit von ought und is (Hume 1739, Buch III, Teil I, Kapitel I) zu verstoßen. Er nimmt ,die ebenso richtige wie in ihrer Bedeutung maßlos überschätzte Tatsache, daß aus Sollsätzen keine Seinssätze deduziert werden können und umgekehrt“ (1968b, 346) zur Kenntnis, verweist jedoch darauf, dass die logische Deduktion eines Satzes aus einem anderen keineswegs der einzige Zusammenhang ist, in dem wissenschaftliche Aussagen zueinander stehen können (vgl. auch 1966a, 113). Für seine Soziologie nicht weniger bedeutsam ist der Zusammenhang problembezogen-funktionaler Aussagen. Aus der Formulierung eines Bezugsproblems lassen sich allein keine einzig richtigen Lösungen ableiten und auch nicht die normative Forderung, das Problem solle (so oder so) gelöst werden. Und doch konstituiert die Formulierung eines Bezugsproblems gemeinsam mit der Angabe einer konkreten Systemreferenz einen Bereich, in dem bestimmte Problemlösungen als mehr oder weniger adäquat bewertet werden können. Daher traut sich Luhmann die Formulierung zu, das Schisma normativer und deskriptiver Forschung und die Forderung der Werturteilsfreiheit seien „durch den Ansatz der funktionalen Systemtheorie überholt“ (1968b, 346). 
Bewertung muss daher an die Wahl einer bestimmten Systemreferenz gebunden werden. ${ }^{9}$,Der Mensch“ etwa als Sammelbegriff für heute bereits mehr als sieben Milliarden Individuen bezeichnet kein empirisch anzutreffendes System und ist daher, das hat Luhmann immer wieder gegen den Humanismus betont, keine für die Systemtheorie mögliche Systemreferenz. Systemtheoretische Kritik kann und will sich nicht „im Namen des Menschen zum Vertreter moralischer Ansprüche“ erklären - sei es in Fragen der richtigen Theorie oder der gerechten Gesellschaft (1978, 28, vgl. 34 f.; 1993a, 35, Anm. 47). Nicht ,der Mensch', nicht ,die Vernunft‘ und auch keine andere Instanz kann ihr dazu dienen, ,,aus absoluten Werten eine Ordnung des Handelns abzuleiten, die jedes System verpflichtet“ (1964b, 396).

Auf ein solches „,soziologisches Naturrecht“ (1965b, 204) als normative Grundlage ist systemtheoretische Kritik allerdings auch gar nicht angewiesen. Gegen eine lange Tradition des Denkens betont Luhmann, dass kein wie auch immer ermittelter Wert Chancen darauf habe, absolute Grundlage systemtheoretischer Wertungen zu sein. Das oft in Werten wie Freiheit oder Gerechtigkeit gesuchte „begründende Absolute“ der Kritik kann für die Systemtheorie nur noch und ausschließlich in der „Funktionsfähigkeit der Systeme“ liegen (1965b, 216), an der gemessen das Maß der Brauchbarkeit eines jeden Wertes (einer jeden Struktur, eines jeden Systems in der jeweiligen Umwelt) zu einer abhängigen Variable wird. Die „Funktionsfähigkeit der Systeme“ zur Grundlage der Kritik zu machen, ohne dadurch den Boden der Systemtheorie zu verlassen, verlangt zugleich die Ablehnung der Vorstellung, dass in dieser Funktionsfähigkeit so etwas wie ein eindeutiges Kriterium der Kritik liegen könnte. Die Systemtheorie betont ja gerade, dass jeder „Systemstandpunkt“ (1968b, 325) notwendigerweise viel zu komplex, vielseitig und widersprüchlich ist, um eine solche Funktion übernehmen zu können. Die Theorie weist darauf hin, dass von dem Standpunkt komplexer Systeme aus gesehen, so gut wie jede Handlung zugleich richtig und falsch, funktional und

\footnotetext{
9 Deshalb muss allerdings nicht ausgeschlossen werden, wertende Urteile auch in Hinblick auf Typen von Sozialsystemen wie Interaktionen und Organisationen zu formulieren oder, etwas konkreter, auf deren Typen wie gesellige Interaktionen, Gerichtsverfahren oder Verwaltungsorganisationen zu beziehen. Auch die Gesellschaft als zugleich besonderes und umfassendes Sozialsystem bleibt eine prinzipiell mögliche Systemreferenz systemtheoretischer Kritik. Indem die Anlage der Systemtheorie und ihr Gedanke der Systemdifferenzierung der durch sie angeleiteten Kritik stets den Wechsel zu übergeordneten Systemreferenzen erlaubt, bietet sie eine Alternative zu einem radikalen Relativismus in normativen Fragen: Was aus Sicht jedes einzelnen Teilsystems einer Organisation oder einer Gesellschaft falsch sein mag, kann sich als das für das Gesamtsystem adäquat erweisen und Rationalität muss in diesem Fall als Zumutung an die Teilsysteme auftreten (und nicht: als Ergebnis der eigenen Einsicht der Teilsysteme in das für das Gesamtsystem Vernünftige).
} 
dysfunktional ist, da Systeme von jeder möglichen Problemlösung in mehrfacher Hinsicht betroffen sind und zwar in der Regel in einigen Hinsichten eher positiv, in anderen eher negativ. Diese Tragik allen Handelns (vgl. 1993c, 229; Kieserling 2014, 94) bekommt die Systemtheorie in den Blick, weil sie Systeme nicht einseitig und strukturalistisch mit ihren Zwecken und sonstigen manifesten Strukturen identifiziert. Stattdessen betont sie, dass der komplexe Systemstandpunkt in diesen Selbstvereinfachungen stets „nur unvollkommen Ausdruck findet“ (1968b, 325; vgl. Kieserling 2014, 90, 95). Deshalb rechnet die Theorie damit, dass ein in Bezug auf die manifesten Strukturen eines Systems konformes Handeln nicht alle „Systembedürfnisse“ hinreichend befriedigen kann. ${ }^{10}$ Neben strukturkonformem Handeln bedarf jedes System auch der Abweichung, dem seinerseits latent gehaltenen und doch strukturierten Normbruch, der sich bei Luhmann in Bezug auf formal organisierte Systeme daher zuweilen den Titel „brauchbarer Illegalität“ verdient (1964b, 304-314).

Das Verständnis sozialer Systeme als wertkomplexer Gebilde mit widerspruchsvollen Systembedürfnissen macht es der Systemtheorie unmöglich, eindeutige Werturteile zu treffen, da ihr kaum eine Problemlösung als ausschließlich ,richtig“ oder ,falsch“ gelten kann. „Systemdienlichkeit“ ist weder eine instruktive Handlungsanleitung, noch ein eindeutiges Kriterium des Richtigen (Kieserling 2012, 136). Mein Argument ist, dass die Systemtheorie dennoch eine Haltung skeptischer Gleichgültigkeit vermeiden kann, der jede mögliche Entscheidung als gleichermaßen gut oder schlecht gilt und die im Sinne der griechischen Tragödie darauf beharrt, dass man es ohnehin nur falsch machen könne. Die zentrale Theorieentscheidung, die ihr stattdessen das Treffen differenzierter Werturteile ermöglicht, betrifft ihre Ziele: Systemtheoretische Wertungen suchen nicht nach in jeder Hinsicht positiven oder gar optimalen, sondern nach brauchbaren Lösungen. Diese Suche bricht mit einer Vorstellung, die sowohl für die auf Aristoteles zurückgehende vormoderne Lehre wahrer Zwecke, als auch für moderne Vorstellungen von Optimierung zentral ist und die sich auch als Annahme einer Exklusivität des Richtigen bezeichnen lässt. Brauchbare Lösungen werden weder durch die immer auch mit ihnen verbundenen Nachteile unbrauchbar, noch dadurch, dass auch andere Lösungsmöglichkeiten den Titel der Brauchbarkeit verdienen. Gesucht werden Lösungen, die ,in einem bestimmten System sinnvoller sind als andere“ (1964a, 42; vgl. 1964b, 308), die sich als vergleichsweise „elegantere

10 Die Rede von ,Systembedürfnissen“ (1964b, 86, 285, 376) oder auch den „Gesamtbedürfnisse[n] des Systems“ (1962,19) ist zumindest beim frühen Luhmann die auffällig anthropomorphe Formel für die oft übersehene Breite der Interessen komplexer Systeme, die in ihrer manifesten Struktur nur sehr selektiv zum Ausdruck kommt. 
Lösungen“ oder als „relativ rationale Entscheidungen“ erweisen (1984, 402f.). Systemtheoretische Kritik ist mithin eine Kritik, die ebenso wie das „normale Leben ... ohne Superlativ“ auskommen kann und muss (1984, 403). In Luhmanns Publikationen der 1990er Jahre findet sich dann eine nochmals andere Formulierung für den hier präsentierten Gedanken, die seine Verbindung mit der Einsicht in die Tragik allen Handelns besonders gut zum Ausdruck bringt. In einer im Wesentlichen der Kritik der (Rechts)Theorie Jürgen Habermas ' gewidmeten Publikation aus dem Jahr 1993 spricht Luhmann von der sinnvollen Möglichkeit, „die Gesellschaft so, wie sie ist und wirkt, zu untersuchen, um Variationsmöglichkeiten $\mathrm{zu}$ finden, die eventuell $\mathrm{zu}$ weniger schmerzlichen Zuständen führen könnten. Könnten!“ (1993d, 56)

In dieser Äußerung werden nicht nur Wirklichkeits- und Möglichkeitssinn der Systemtheorie miteinander verbunden, ${ }^{11}$ Luhmann gibt in ihr auch eine im Spätwerk verstärkt auftauchende Formel für das Ziel systemtheoretischer Kritik aus: Die Suche nach ,weniger schmerzlichen Zuständen‘. Diese Suche akzeptiert, dass keine Strukturwahl, keine Problemdefinition, keine Zwecksetzung, keine Selbstvereinfachung (sozialer) Systeme die Aussicht auf Wahrheit, auf exklusive Richtigkeit hat. Sie weist davon ausgehend darauf hin, dass jede Selbstvereinfachung dennoch darauf befragt werden kann, ob sie vergleichsweise gut oder schlecht funktioniert und ob sie aus diesem Vergleich mit dem Anspruch heraustreten kann, „eine weniger schmerzhafte, besser erträgliche Konstruktion“ (1996, 45) zu sein. In einem Edmund Husserl gewidmeten Vortrag sympathisiert Luhmann in Anlehnung an eben diesen Philosophen mit einer als selbstkritisch und ironisch bezeichneten Form der Vernunft. Ihre „Bewährungsproben“ habe sie in nichts anderem als ,in der Therapie, die weniger schmerzhafte Lösungen zu erreichen versucht“ (1996, 45; vgl. für ähnliche Formulierungen 1992, 141 f.). ${ }^{12}$

Eine so verstandene systemtheoretische Kritik denkt die Eignung von Problemlösungen nicht als zweiwertige Unterscheidung, sondern als Kontinuum. Sie weigert sich, Kritik ,wie in Frankfurt ... als emphatische Ablehnung des Gegenstandes der Kritik“ zu verstehen (1996, 25) und begreift sie stattdessen als Mitar-

11 Diese Verbindung scheint mir den Anspruch und die Fähigkeit der Systemtheorie, wertende und beschreibende Soziologie miteinander zu verbinden, gut zu charakterisieren. Ich komme am Ende des Textes darauf zurück.

12 Peter Sloterdijk zählt zu den wenigen Autoren, die auf die wertenden und ,therapheutische[n] Konsequenzen“ (Sloterdijk 2010, 136) der Luhmannschen Systemtheorie aufmerksam gemacht haben. In einer Formulierung, die derjenigen von Kritik als Suche nach weniger schmerzlichen Zuständen sehr ähnlich ist, hält Sloterdijk den „Austausch eines unlebbaren Realitätskonstruktes gegen ein weniger unerträgliches“ für die Perspektive einer an Luhmann orientierten Sozialkritik (152). 
beit an der kritisierten Sache. Systemtheoretische Kritik will also eine zumindest potentiell hilfreiche Kritik sein und sie kann dies nur sein, wenn sie eine faire Kritik ihres Gegenstandes ist. Statt ihn mit den (möglicherweise soziologisch und praktisch naiven) Idealen des Kritikers zu konfrontieren, erforscht sie zunächst seine wirklichen Möglichkeiten der Selbstvariation und kann daher mit ihnen rechnen.

\section{Die Sprache der Probleme und ihrer Lösungen - Äquivalenzfunktionalismus als Methode deskriptiver und normativer Soziologie}

Die Ziele systemtheoretischer Kritik liegen also in der Suche nach brauchbaren oder „weniger schmerzhaften“ Problemlösungen für konkrete Systeme. Die Form dieser Kritik ist die Ermittlung von Systemproblemen und der auf Systemdienlichkeit zielende Vergleich möglicher Lösungen. Ihre Besonderheit ist, dass sie in beiden Hinsichten danach strebt, die Selbstverständlichkeiten der wirklichen Wirklichkeit und der in ihr Handelnden zu problematisieren. Eine so verstandene Kritik bedient sich der Methode der Systemtheorie, die Luhmann unter der Bezeichnung Äquivalenzfunktionalismus entwickelt hat. Im Zuge dieser Entwicklung beruft sich Luhmann auf die gleichen Motive, die auch seine Kritik am Postulat der Werturteilsfreiheit tragen: Die „Aussicht auf eine methodisch-einheitliche Sozialwissenschaft“ $(1962,9)$ und das Selbstbewusstsein, die Diskrepanzen zwischen soziologisch überzeugender und wissenschaftstheoretisch erlaubter Forschung zu Ungunsten der Wissenschaftstheorie zu entscheiden (1962, 13; 1974).

Am Anfang jeder äquivalenzfunktionalistisch durchgeführten Analyse steht die Konstruktion eines Bezugsproblems als Eigenleistung der Theoretikerin. Dieses Bezugsproblem dient der Analyse als „regulatives Sinnschema, das einen Vergleichsbereich äquivalenter Leistungen organisiert“ (1962, 14) und in eben dieser „Eröffnung eines (begrenzten) Vergleichsbereichs“ (13) liegt der Sinn der funktionalen Analyse als einer „Abstraktions und Vergleichstechnik“ (15; vgl. auch 1964a, 37; 1974). Das „Beobachtungsschema“ des funktionsorientierten Vergleichs bleibt auch in späteren Werkphasen die Methode der Systemtheorie (1984, 468), und Abstraktion, die Eröffnung von Vergleichshorizonten im Unterschied zur Erklärung der konkreten Wirklichkeit, gilt weiterhin als „Leitfrage“ und primäres Erkenntnisziel systemtheoretischer Soziologie (1984, 395). So gut bekannt all dies zumindest unter Systemtheoretikerinnen sein dürfte, so wenig scheint mir zumeist deutlich zu werden, dass der Äquivalenzfunktionalismus 
nicht nur als Methode soziologischer Beobachtung, sondern auch soziologischer Kritik gelesen werden kann und muss. Zwar ist bereits vereinzelt darauf hingewiesen worden, dass der Äquivalenzfunktionalismus kritikaffine Züge trägt (Kneer 1996, 395 f.; Tacke/Kette 2015, 242 ff.). Dass er nur als wertendes Verfahren, als vergleichende „Ermittlung und Bewertung von Alternativen“ (1968b, 28, meine Hervorhebung) angemessen verstanden werden kann, wird jedoch soweit ich sehe bislang nicht hinreichend betont. ${ }^{13}$

Jede Form normativer Sozialkritik steht vor dem Problem, den Maßstab ihrer Kritik begründen zu müssen (vgl. Greve 2015). Um dem Vorwurf der Beliebigkeit und des Paternalismus der eigenen Kritik zu entgehen, halten es viele Ansätze der Sozialkritik für notwendig, die durch Handelnde selbst formulierten Zwecke, Probleme, Werte oder ihre institutionalisierten Handlungsnormen ohne weitere Kommentierung als Ausgangspunkte und Maßstäbe der Kritik zu übernehmen. Eine so vorgehende Kritik - etwa die konditionale Kritik bei Max Weber oder die immanente Kritik in der Kritischen Theorie (s. o.) - neigt stets dazu, eine Wiederholung der im Gegenstandsbereich bereits formulierten oder doch formulierbaren Selbstkritik zu sein. In Abgrenzung dazu traut sich Luhmanns Äquivalenzfunktionalismus auch die Problematisierung wirklicher Problemdefinitionen zu. Die Methode zielt nicht nur darauf ab, ,jede Problemlösung“, sondern auch ,,jede Problemstellung zu hinterfragen, auf grundsätzlichere Probleme aufzulösen, letztlich also alle Evidenz bis hin zu der Welt selbst in ein Problem zu verwandeln, in Hinblick auf welches Problemlösungen verglichen werden können“ (1967a, 119). ${ }^{14}$ Luhmann ist davon überzeugt, dass es sich lohnt, „die Problemfindung selbst zum Problem zu machen“ $(1971,206)$ und den ersten Schritt einer Soziologie in kritischer Absicht mithin darin zu sehen, „Probleme anders und im Hinblick auf neuartige Lösungen zu formulieren“ (1997, 1133; vgl. 1986, 17, 162). Systemtheoretische Kritik gewinnt ihre Ausgangspunkte und Maßstäbe also weder aus einem Schatz tradierter Werte noch aus den Selbstverständlichkeiten oder

13 Damit widerspreche ich nicht Feststellungen wie derjenigen Harry M. Johnsons aus den 1960er Jahren, ,funktional' meine keineswegs ,gut‘ und ,dysfunktional' keineswegs ,schlecht‘ (Johnson 1961, 77). Diese Feststellung ist richtig, so lange die Worte ,gut' und ,schlecht‘ wie bei Johnson in einem nicht auf bestimmte Bezugsprobleme oder Bezugssysteme relativierten Sinn verwendet werden. Meinem Hinweis, ,funktional für meine, gut für', dürfte auch er nicht widersprochen haben.

14 Die Grenzen der ihr möglichen Problematisierung will die Theorie dabei selbst bestimmen. Diese Grenzen sind bei Luhmann solche der theoretisch kontrollierbaren Komplexität und der Verantwortlichkeit von Sozialkritik für ihre Folgen. Ich komme abschließend kurz darauf zurück. 
Üblichkeiten des kritisierten Gegenstandes. Schon in die Ermittlung der Bezugsprobleme der Kritik soll die inkongruente Perspektive der Soziologie eingehen. ${ }^{15}$

Die von mir vorgeschlagene Lesart der Systemtheorie betont, dass die Sprache dieser Theorie die „Sprache der Probleme und ihrer Lösungen“ (1967b, 75) ist und sie sieht den zweiten Schritt systemtheoretischer Kritik in dem wertenden Vergleich wirklicher und möglicher Problemlösungen. Der diesen Vergleich tragende Gedanke ist, die Rationalität eines Systems, etwa einer Organisation, nicht in dem voraus zu setzen, „was es seinem Wesen nach unter Ausschluß anderer Möglichkeiten ist, sondern nur in der Ordnung seiner Potentialitäten, in der Verfügbarkeit anderer Möglichkeiten, die einen gegebenen Zustand funktional äquivalent ersetzen können, wenn die Umwelt sich ändert - also gerade in der Unabhängigkeit von einem bestimmten konkreten Sein“ (1966a, 110 f.). Dem Vergleich in kritischer Absicht geht es um die Analyse vorhandener Systeme als eines „Spielraums“ (1965b, 213), in dem vieles, aber keineswegs beliebiges sinnvoll möglich ist. Stets ist dabei die soziologisch bestimmte „Funktion das Schema, nach dem sich Ermittlung und Bewertung von Alternativen richten“ (1968b, 28, meine Hervorhebung). Äquivalenzfunktionalistische Kritik als „neue Vernunft des Vergleichens“ (1968b, 49; 1965b, 8) „stimuliert die Suche nach Alternativen. Sie vermag so die zähe Konservativität des natürlichen Erlebens für Neurungen aufzuschließen, indem sie in je spezifischen Hinsichten kontrollierbare Varationsmöglichkeiten eröffnet“ (1968b, 29; vgl. für eine in Teilen ähnliche Lesart der Systemtheorie Opitz 2013, 42, 59).

Äquivalenzfunktionalistische Vergleiche ermitteln und bewerten mögliche Problemlösungen in Hinblick auf spezifische Bezugsprobleme. Eine so vorgehende systemtheoretische Kritik weiß darum, dass (soziale) Systeme wertkomplexe Systeme sind, die verschiedene Probleme lösen müssen, wobei eine in bestimmter Hinsicht sinnvolle Lösung in anderen Hinsichten oft Folgeprobleme nach sich zieht. Der systemtheoretischen Kritikerin ist es dadurch unmöglich, potentielle Problemlösungen in Hinblick auf nur eine (etwa: die primäre, manifeste) Systemfunktion zu beurteilen. Ich habe bereits darauf hingewiesen, dass systemtheoretische Wertungen dadurch keineswegs ausgeschlossen werden, sondern lediglich schwerer zu treffen sind und ihrem Charakter nach ambivalenter ausfallen müssen. Betont werden muss, dass systemtheoretische Kritik

15 Die Formulierung von Bezugsproblemen als Eigenleistung systemtheoretischer Analyse und Kritik erfolgt freilich keineswegs beliebig, sondern in Abstimmung mit der Theorie und sie kann ihrerseits auf ihre Rationalität befragt werden. Die Diskussion der dafür von Luhmann vorgeschlagenen Kriterien wäre das Thema eines eigenen Textes, siehe als Ausgangspunkte etwa 1962, 14, 19, 27; 1964a, 37 f., 47; 1967a, 118; 1968b, 49, 157, 312, 326 f., 333. 
sich daher oft nur schlecht zur Mobilisierung von Protest und Empörung eignen dürfte. ${ }^{16}$ Widersprochen werden muss dagegen der verbreiteten Vorstellung, (äquivalenz)funktionalistische Kritik sei darauf beschränkt, innerhalb vorgegebener Möglichkeitsräume (Systeme) nach immer brauchbareren Lösungen zu suchen, könne diese Möglichkeitsräume selbst jedoch nicht problematisieren. Auch dazu ist eine an Luhmann orientierte systemtheoretische Kritik in der Lage. Ihrer „Unzufriedenheit mit den Problementscheidungen“ des jeweils untersuchten (Teil)Systems kann sie Ausdruck geben „durch Aufstieg zu grundsätzlicheren Problemen“ oder umfassenden Systemreferenzen (1966a, 117; vgl. Kieserling 2014). Alles kann sie problematisieren, aber nicht alles zugleich, und nur dann, wenn sie es kann. ${ }^{17}$ Jede ihrer Problematisierungen muss von einem anzugebenden Ort her erfolgen und diesen Ort, diese Menge wirklich gewählter Problemlösungen, muss sie zwar für den Moment der Analyse, nicht aber schlechthin als seinerseits unproblematisch behandeln.

Die hier vorgeschlagene Deutung des Äquivalenzfunktionalismus als soziologisch kontrollierter Erzeugung und Bewertung alternativer Problemformulierungen und Problemlösungen lässt sich durch den Hinweis ergänzen, dass der funktionsorientierte Vergleich nicht nur die Methode der Systemtheorie, sondern zugleich auch ein geeignetes Schema zur praktischen Gestaltung der Sozialwelt ist. Denn „Problemlösung erfordert im Denken und Handeln gleichermaßen

16 Darin liegt wiederum ein Hinweis, dass soziologisch begründete Wertungen die Autonomie des Fachs ganz im Gegensatz zu den Befürchtungen Webers $(1919,31)$ steigern. Systemtheoretische Kritik vermag die Problemlasten zu übernehmen, die von einer wertfreien oder lediglich konditional bzw. immanent kritisierenden Soziologie getragen werden. Die Willkür des Wertens wird nicht durch ein Verbot von Wertungen, sondern durch deren fachliche Disziplinierung zu erreichen versucht (vgl. in diesem Sinne auch 1904, 20). Nur durch eine solche Selbstdisziplinierung kann es soziologischer Kritik auch gelingen, überraschende, gegenüber der Selbstkritik des Gegenstandsbereiches distanzierte, Wertungen hervorzubringen. Diesen von ihr aus theoriesystematischen Gründen nur schwer erfüllbaren Anspruch scheint die zeitgenössische immanente Kritik mittlerweile aufgegeben zu haben. Titus Stahl zufolge weiß die immanente Kritikerin schon vor aller Analyse, wie Lob und Tadel zu verteilen sind: „die Strategie der immanenten Kritik dient daher nicht primär dazu, die richtigen Normen zu finden, sondern nachzuweisen, dass bestimmte, von den Kritikerinnen und Kritikern akzeptierte Normen eine Basis in der sozialen Realität haben“ (Stahl 2014, 54). Systemtheoretische Kritik will und kann dagegen allenfalls zufälligerweise die nachträgliche Legitimation anderweitig, etwa in Protestbewegungen, erworbener oder in politischen Ideologien kombinierter Werthaltungen sein. Insofern ist sie ebenso wie die ihr zu Grunde liegende (äquivalenz)funktionalistische Methode ideologisch neutral im Sinne Robert K. Mertons (1957, 17-42).

17 Wenn sie es kann, das heißt: Wenn sie die dadurch ,wachsende Komplexität der Folgeprobleme ins Auge fassen und verarbeiten“ (1966a, 117) kann. Ich komme abschließend kurz auf diese Selbstbestimmung der Grenzen rationaler Sozialkritik in der Systemtheorie zurück. 
Orientierung an Alternativen“ und das Erkenntnisziel der Systemtheorie liegt in nichts anderem als der „Kontrolle von Alternativen; zunächst die theoretische Kontrolle und dann, im Rahmen des Möglichen, die praktische Kontrolle“ (1964a, 35 f.). Nicht nur die Grenzen zwischen normativer und deskriptiver Soziologie, auch die Grenzen von soziologischer Theorie und außersoziologischer Praxis können durch eine an Luhmann orientierte Systemtheorie und die ihr zugehörige Methode überbrückt werden, ohne die Differenzen dieser Welten zu übersehen:

Der Erkenntnisgewinn durch Vergleich ist für die theoretische wie für die praktische Orientierung die gleiche. Die Methodik der Abstraktion von Bezugsgesichtspunkten und der Ermittlung von Alternativen im Rahmen von Äquivalenzserien ist die gleiche, mag sie von heuristischen oder von strategischen Motiven getragen sein. Und die typischen ,Kosten der Abstraktion: daß ihre Einsichten unter verschobenen Bezugsgesichtspunkten irrelevant werden, sind die gleichen. Nur die Gründe für die Wahl jener Bezugsgesichtspunkte differieren. Die Wissenschaft begründet ihre Wahl aus dem Zusammenhang einer Theorie, die Praxis durch Bezug auf Werte (...) Durch Austausch dieser Gründe, durch Übergang von theoretischer zu wertmäßiger Begründung, kann die Praxis sich der wissenschaftlichen Erkenntnis bemächtigen, ohne daß deren Wahrheitsgehalt dadurch verfälscht würde (1964a, 47). ${ }^{18}$

\section{Der soziologisch disziplinierte Möglichkeits- sinn der Systemtheorie - Systemtheoretische Kritik als problemloyale Kritik}

In diesem Beitrag habe ich gezeigt, dass Niklas Luhmann die Formel der Werturteilsfreiheit zur Selbstbeschreibung der Systemtheorie abgelehnt hat. Er verstand seine Theorie als Grundlage für eine deskriptive Betrachtung und soziologisch disziplinierte Bewertung sozialer Systeme. Eine an Luhmann anschließende Selbstverortung systemtheoretischer Kritik als einem Potential der Systemtheorie scheint mir mit Hilfe der von Kieserling (2004b) explizierten Unterscheidung der

18 Auch in späteren Schriften betont Luhmann, dass die soziologische Vergleichstechnik auf einem gemeinsamen „Kontinuum des Vergleichsinteresses“ mit alltagsweltlichen Vergleichen liegt. Auf diesem Kontinuum sind soziologische Vergleiche dann jedoch unter anderem zeitaufwendiger, vielseitiger und von der manifesten Struktur des jeweiligen Systems aus gesehen abseitiger angelegt (1990, 408 f.; 1974, 17). Diese Perspektive auf das Verhältnis von soziologischer Theorie und außersoziologischer Praxis betont sowohl ihre Differenz, als auch die Möglichkeit ihrer wechselseitigen Verständigung (vgl. dazu auch etwa o.J., 228; 1962, 15 ff.; 1966a, 105; 1967b, 75; 1968b, 236, 347 ff.; 1971, 240). 
Selbstbeschreibung sozialer Systeme von ihrer Fremdbeschreibung gut vollzogen werden zu können. Gemäß dieser Unterscheidung gelten beispielsweise die von Pädagogen angefertigten Theorien als „mehr oder minder systemloyale Sicht“ auf Organisationen und Situationen erzieherischen Handelns und werden dem Erziehungssystem als dessen eigene Reflexionstheorie zugerechnet. Die Soziologie der Erziehung gilt dagegen als der Wissenschaft zugehörige und „mehr oder minder respektlose Sicht“ auf denselben Gegenstand (Kieserling 2004b, 88).

Meine These ist, dass sich systemtheoretische Kritik einer exklusiven Gegenüberstellung von respektloser Fremdbeschreibung und systemloyaler Selbstbeschreibung entzieht. Jenseits dieser Unterscheidung ist sie dadurch charakterisiert, distanziert und loyal zugleich vorzugehen. Distanziert zeigt sich der externe Blick systemtheoretischer Kritik gegenüber den manifesten Strukturen, den Selbstvereinfachungen und Selbstverständlichkeiten des kritisierten Systems. Mit dem soziologisch gebildeten Bezugsproblem legt er „verfassungsexterne Maßstäbe“ an das System an, das er zu kritisieren sucht (1965b, 209). Will die Kritik eine nicht beliebige, sondern disziplinierte Respektlosigkeit sein, kann sie auf eine gewisse Loyalität gegenüber dem beobachteten und kritisierten System jedoch nicht verzichten. Ihr externer Blick muss sich loyal zeigen in Bezug auf soziale Systeme als eines „Möglichkeitsspielraums“ (1966a, 114; 1984, 388). Diese systemloyale Kritik zielt darauf ab, soziale Systeme als wirkliche Möglichkeitsräume zu erforschen, sie also auf die Bedingungen und Grenzen der ihnen möglichen Selbstvariation zu befragen, um so das „Maß unserer Möglichkeiten“ zu bestimmen (1966b, 139; vgl. 1966a, 115).

Die systemtheoretische Kritikerin ist dabei zwar nicht an das Selbstverständnis der Kritisierten gebunden, wohl aber an deren mögliche Wirklichkeiten (wirkliche Möglichkeiten). Das Anliegen ihrer systemloyalen Kritik ist es also durchaus, sich auf „Linien [zu] bewegen, auf denen Verhalten zur Problemlösung beitragen könnte“ $(1990,697)$ - unter diesen Linien können aber auch solche sein, die in der Selbstbeschreibung des Gegenstandes nicht vorgesehen sind und daher von der Kritikerin erst erzeugt werden müssen. Mit einer anderen Formulierung lässt sich auch sagen, dass systemtheoretische Kritik dem ,prinzipiellen Verdacht der Illoyalität, ja des Verrats“, den jede Kritik auf sich zieht (Lepsius 1964, 76), auf doppelte Weise begegnet. Einerseits bekennt sie sich der Illoyalität (Distanz) gegenüber jeder einzelnen wirklichen und möglichen Problemlösung schuldig. Andererseits versichert sie zugleich, diesen Verrat nur auf Grundlage einer (zeitweisen) Loyalität gegenüber dem Bezugsproblem (Problemsystem) ihrer Kritik zu 
begehen. Systemtheoretische Kritik ist in diesem Sinne auch eine problemloyale Kritik. ${ }^{19}$

Luhmann hat stets Wert darauf gelegt, systemtheoretische Kritik nicht nur als problemorientierte und problemloyale, sondern auch als verantwortliche Form der Sozialkritik zu präsentieren: „Die Reverenz, die der Soziologe der Geschichte, das heißt der schon reduzierten Komplexität, zu erweisen hat, läßt sich mithin in einer einzigen Formel für die Praxis ausdrücken: ,Nichts zu ändern, es sei denn, daß dem zu ändernden Zustand all seine Funktionen abgetauscht werden können.““ (1967b, 85, vgl. 70; 1969, 256; 1993a, 503) Äußerungen dieser Art sind oft als Ausdruck einer wertkonservativen Haltung der Systemtheorie missverstanden worden. Ältere (Beck/Beck-Gernsheim 1994, 26 ff., 36) und neuere (Scherr 2015, 33) Stimmen haben ihr vorgehalten, ihr Möglichkeitssinn sei zugunsten ihres Wirklichkeitssinns verkümmert. Der voranstehende Text führt zu einer anderen Diagnose: Die Suche nach einer selbstdisziplinierten Sozialkritik zeigt, dass die Systemtheorie in sich kombinieren kann, was Robert Musil Wirklichkeits- und Möglichkeitssinn nannte. Die Wirklichkeit, der Status Quo, wird von ihr detailliert zu beschreiben und zu erklären versucht, erscheint ihr deshalb aber nie als prinzipiell alternativlos oder gar perfekt. Die wirkliche Wirklichkeit kann aus einer systemtheoretischen Perspektive jederzeit als „Aufgabe und Erfindung“ behandelt werden, indem ihr entgegengehalten wird: „Nun, es könnte wahrscheinlich auch anders sein“ (Musil 1930, 16).

Zugleich erzieht eine an Luhmann orientierte Systemtheorie dazu, den Status Quo und die in ihm bereits gelösten Probleme würdigend zu betrachten. Durch diese nur auf den ersten Blick widersprüchliche Ausrichtung lenkt systemtheoretische Kritik die Aufmerksamkeit auf die Frage, wie genau die Wirklichkeit anders sein könnte, ohne schlechter geworden zu sein. Anders gesagt: Die beiden methodischen Annahmen, dass es stets Gründe dafür gibt, „daß die Dinge so sind wie sie sind“ (1993b, 171) und das zugleich alles anders sein könnte, als es ist, ergänzen sich in der Systemtheorie, anstatt sich auszuschließen. Die Funktionen wirklich gewählter Problemlösungen anzugeben, heißt für diese Theorie nichts anderes, als die Bedingungen ihrer Ersetzbarkeit zu benennen (1965b, 8; 1968a, 22). Ein anspruchsvoller Möglichkeitssinn im Sinne Musils, eine an der Realität geschulte

19 Der Begriff des „systemloyalen Kritikers“ wird von André Kieserling (2015) genutzt, aber nicht weiter ausgeführt. Das mögliche Missverständnis, dieser Begriff empfehle systemtheoretischer Kritik eine konservative, auf die Bewahrung bestehender Strukturen zielende Haltung, mag vermieden werden, wenn nicht von Systemloyalität, sondern von Problemloyalität der Kritik gesprochen wird, mit dem Zusatz, dass jedes Problem(system) in weiteren Analysen seinerseits problematisiert werden kann. 
und über sie informierte Phantasie, vermeidet damit den Utopismus, das bloße Wünschen, dessen Resultate nur „müßige Hirngespinste bedeuten“ und deshalb zu enttäuschten Hoffnungen führen müssen, weil er ein disziplinierter Sinn für mögliche Wirklichkeiten ist (Musil 1930, 17). Dieser in Theorie und Methode der Luhmannschen Systemtheorie angelegte pragmatische Möglichkeitssinn kann in seinen Ergebnissen dann auch einen Beitrag zu der von C. Wright Mills geforderten Steigerung sozialer Freiheiten durch soziologische Phantasie leisten: „Freiheit ist nicht nur die Möglichkeit, zu tun, was man will; auch nicht bloß die Gelegenheit, zwischen Alternativen zu wählen. Freiheit ist vor allem die Möglichkeit, die verfügbaren Alternativen zu formulieren und über sie zu streiten - und dann eine Wahl zu treffen.“ (Mills 1963, 260)

Das systemtheoretische Lob der Wirklichkeit ist mithin kein Ergebnis ihrer Kritik der Sozialwelt, sondern deren Anfang (1968a, 26, 22) ${ }^{20}$ Es sollte als methodisches Lob verstanden werden, als Suche nach dem Maß, an dem jeder Änderungsvorschlag gemessen werden muss. Je größer das Lob der Wirklichkeit, desto schwerer und desto besser die an ihr mögliche Kritik. Auch in diesem Sinne ist soziologische Aufklärung als Abklärung der Aufklärung selbst Teil der Aufklärung (1967b, 67). Luhmanns Kritik der Kritiker muss nicht als Selbstzweck, sie kann als Mitarbeit an einer soziologisch informierten Selbstkritik der modernen Gesellschaft und der Sozialsysteme in ihr verstanden werden.

\section{Die Grenzen systemtheoretischer Kritik}

Ich habe bereits betont, dass eine soziologisch informierte Kritik der sozialen Welt gerade aus einer systemtheoretischen Perspektive nicht als die einzig richtige oder wünschenswerte Form der Kritik angesehen werden kann. Um dem möglichen Eindruck einer Selbstüberschätzung soziologisch informierter Kritik

20 Nicht ein Konservatismus im Sinne Karl Mannheims (1927, $411 \mathrm{ff}$.) als prinzipielle Bejahung und Verteidigung des Bestehenden charakterisiert die Theorie Luhmanns, sondern ein „unfreiwillige[r] Konservatismus aus Komplexität“ (1968a, 19). Wird die zu kritisierende Wirklichkeit zu komplex, um die Folgen der Variation dieser Wirklichkeit überblicken zu können, versagte sich Luhmann das wertende Urteil über sie oder betonte alternativ die Unsicherheit des Urteils. Im Übrigen spricht viel dafür, dass die Orientierung am Status Quo rationale Kritik erleichtert. Dank dieser Orientierung muss sich die von der Kritikerin gegebenenfalls vorgeschlagene Änderung nicht als allen möglichen Lösungen überlegene, optimale Lösung präsentieren, sondern lediglich (aber auch dies ist in Bezug auf wertkomplexe Systeme oft sehr viel verlangt) als der jeweils aktuell gewählten Lösung überlegen. 
entgegenzuwirken, seien abschließend und zusammenfassend drei wichtige Grenzen systemtheoretischer Kritik benannt.

Erstens ist die Reichweite systemtheoretischer Kritik stets auf konkrete Sozialsysteme begrenzt. Wertende Urteile ohne Angabe einer Systemreferenz gehören ebenso wenig zu den Möglichkeiten systemtheoretischer Kritik wie die Identifikation des funktional Sinnvollen mit dem moralisch Richtigen. Die in kritischer Absicht betrachteten sozialen Gebilde werden in der Systemtheorie zwar ebenso wie in der von Rahel Jaeggi kürzlich vorgelegten Version immanenter Sozialkritik als „Problemlösungsinstanzen“ (Jaeggi 2014, 141), als „Inkarnation kontingent gelöster Probleme“ $(1974,18)$ begriffen. Einig mit Jaeggi ist sich systemtheoretische Kritik auch darin, „Funktionieren und Nichtfunktionieren“ zu den normativen Kriterien einer bewertenden Kritik der in diesen sozialen Gebilden ablaufenden, unabschließbaren Problemlösungsprozesse zu erheben (Jaeggi 2014, 289 f., $175 \mathrm{ff}$.). Aus diesen Theorieentscheidungen folgt für systemtheoretische Kritik anders als für die immanente Kritikerin Jaeggi jedoch keineswegs eine Identifikation von ,funktionalen' und ,ethischen“ Normen (so Jaeggi 2014, 175). Systemtheoretische Kritik teilt nicht die Auffassung, dass die „letzten idealen Bezugspunkte“ der Kritik „für die Sozialordnung ebenso wie für die Einzelperson ,gut sein“ müssten (1964b, 395; vgl. 1978, 32).

Die für systemtheoretische Kritik unverzichtbare Wahl einer Systemreferenz führt zweitens zu einer Grenze der Begründbarkeit dieser Form der Sozialkritik. Die Wahl eines Problemsystems, gegenüber dem sich eine systemtheoretische Kritikerin im Rahmen der jeweiligen Analyse loyal verhält, kann von der Theorie zwar angeleitet, aber nicht übernommen werden. Es bleibt eine Wahl, die eine Kritikerin auf Grundlage von Werten zu treffen hat - seien es die Werte der Wissenschaft (Anschlussfähigkeit an wissenschaftliche Kommunikationen), einer außerwissenschaftlichen Praxis oder die Werte der Kritikerin selbst, die mehr oder weniger identisch sein können mit den Werten der Gesellschaft, in der sie lebt. Als eine nur (system)relativistische Kritik wäre systemtheoretische Kritik dennoch unvollständig charakterisiert. Der Gedanke der Systemdifferenzierung legt in vielen Fällen nahe, das jeweils umfassende System (und sei es das der Gesellschaft, etwa in Fragen ihrer ökologischen Selbstgefährdung) als Systemreferenz besonderen Rechts zu behandeln.

Die Begrenzung der Reichweite systemtheoretischer Kritik auf konkrete Systemreferenzen und die Grenzen der rationalen Begründbarkeit der jeweiligen Wahl einer Systemreferenz werden drittens durch Grenzen der Eindeutigkeit systemtheoretischer Kritik ergänzt. Das Verständnis sozialer Systeme als wertkomplexer Problemlösungszusammenhänge führt dazu, dass die wertenden Aussagen der Systemtheorie in der Regel nicht durch Eindeutigkeit auffallen, sondern von Ambivalenz geprägt sind. In vielen Fällen dürfte eine auf Wirksamkeit abzie- 
lende Sozialkritik deshalb gut damit beraten sein, auf systemtheoretische Fundierungen zu verzichten.

Während aktuelle Ansätze der ,Kritischen Systemtheorie“ die „normative Wendung der Systemtheorie“ (Fischer-Lescano 2013, 15) fordern, habe ich in dem vorliegenden Text darauf hingewiesen, dass und inwiefern bereits die von Luhmann vorgelegte Systemtheorie auch als wertende Kritik der sozialen Welt (sozialer Strukturen, Semantiken, Beobachtungsweisen) verstanden werden muss. ${ }^{21}$ Bei der Bearbeitung der Frage nach der Methodologie systemtheoretischer Wertungen und deren theorieimmanenter Grenzen habe ich auf den Vorschlag der Fusion der Systemtheorie mit anderen, als ,kritisch“ geltenden, theoretischen Paradigmen verzichtet. Diese auch in der Diskussion um ,Kritische Systemtheorie‘ üblichen Vorschläge scheinen mir nicht nur zu einem Verlust theoretischer Konsistenz zu führen (so auch Bora 2016, 265 f.), sondern auch zu dem Verzicht auf den Anspruch und Reiz systemtheoretischer Kritik, ihren wertenden Möglichkeitssinn nicht durch die Bejahung bestimmter Werte zu disziplinieren. Kein Aspekt der sozialen Welt gilt systemtheoretischer Kritik als schlechthin unproblematisierbar, weder die expliziten Wertsetzungen der Handelnden (Weber, interne Kritik), noch die von ihnen implizit unterstützten Normen (zeitgenössische immanente Kritik). Auf die Fundierung der Kritik in bestimmten Werten wie dem sozio-

21 In dem hier gewählten Zugang zu dem wertenden Potential der Luhmannschen Theorie habe ich (auch) aus Platzgründen auf eine Diskussion der von Luhmann selbst durchgeführten wertenden Analysen verzichtet. Siehe für einige von vielen Beispielen einer problemorientierten, von der Wirklichkeit ausgehenden und sie dann in Bezug auf Problemdefinitionen und Problemlösungen transzendierenden Kritik etwa Luhmanns Bemerkungen zu einer „sinnvolle[n] Kritik der öffentlichen Meinung, die eine Gesellschaft jeweils hervorbringt“ (1970b, 33), seine Kritik (wertende Diskussion) der werthaltigen Sprache in der Politik anhand eines Parteiprogramms der CDU (1977), seine Unterscheidung funktionaler von ,merkwürdigen' Skandalisierungen in der Politik (1993b, 173), seine „Kritik der persönlichen Schadenshaftung“ in der öffentlichen Verwaltung (1966b, 113) oder die von ihm formulierten Ausgangspunkte zu einer „strukturellen Kritik des Preismechanismus“ als zentraler Struktur des Wirtschaftssystems (1988, 40).

Gemäß der hier vertretenen These prägt diese Form funktionalistischer Sozialkritik das Gesamtwerk Luhmanns und eben deshalb kombiniert eine Vielzahl seiner Schriften beschreibende Analysen mit oft (scheinbar) beiläufig bewertenden Ausführungen zu ihrem jeweiligen Gegenstand. Ein wichtiger Teil der von Luhmann formulierten Kritik besteht auch in der Kritik institutionalisierter und somit sozial wirkmächtiger semantischer Traditionen. Ich denke etwa an seine Kritik der Rationalitätssemantik (o.J. [1982]), seine hier in Ansätzen rezipierte Kritik der Vorstellung, gelungene Sozialität sei am Ideal der Widerspruchsfreiheit zu messen und natürlich auch an die Kritik der Forderung einer werturteilsfreien Sozialwissenschaft, die ich im ersten Teil dieses Textes rekonstruiert habe. Mir ging es in diesem Text jedoch nicht um eine systematische Untersuchung der von Luhmann gewählten Anwendungsfälle systemtheoretischer Wertungen, sondern um eine Rekonstruktion der hinter diesen Wertungen stehenden Methodologie. 
logisch gesehen normativ einseitigen Ideal der Widerspruchsfreiheit sozialer Gebilde kann und muss sie verzichten. Stattdessen versteht systemtheoretische Kritik die Brauchbarkeit jeder Problemlösung als eine systemrelative Variable. Loyal zeigt sich systemtheoretische Kritik allein gegenüber den wirklichen Möglichkeiten sozialer Systeme, die sie als Problemsysteme begreift. Die Form dieser Kritik liegt in einem auf Problemformulierungen und Problemlösungen bezogenen und dabei soziologisch informierten „Hinweis auf ein Nichtausschöpfen des Möglichen“ (1990, 695).

Danksagung: Für hilfreiche Kommentare zu früheren Fassungen des Textes danke ich den Herausgebern dieses Themenschwerpunktes, einem anonymen Gutachter und dem Redakteur dieser Zeitschrift sowie meinen Kolleginnen und Kollegen an der Bielefelder Fakultät für Soziologie.

\section{Literatur}

Adorno, Theodor W. (1972): Gesellschaft (I), in: ders., Soziologische Schriften, Bd.1. Frankfurt a. M.: Suhrkamp, 8-19.

Amstutz, Marc (2013): Der zweite Text. Für eine Kritische Systemtheorie des Rechts, in: Marc Amstutz/Andreas Fischer-Lescano (Hrsg.), Kritische Systemtheorie. Zur Evolution einer normativen Theorie. Bielefeld: transcript, 365-401.

Amstutz, Marc/Fischer-Lescano, Andreas (Hrsg.) (2013): Kritische Systemtheorie. Zur Evolution einer normativen Theorie. Bielefeld: transcript.

Beck, Ulrich/Beck-Gernsheim, Elisabeth (1994): Individualisierung in modernen Gesellschaften - Perspektiven und Kontroversen einer subjektorientierten Soziologie, in: dies., Riskante Freiheiten. Individualisierung in modernen Gesellschaften. Frankfurt a. M.: Suhrkamp, 10-39.

Bittner, Rüdiger (2009): Kritik, und wie es besser wäre, in: Rahel Jaeggi/Tilo Wesche (Hrsg.), Was ist Kritik? Frankfurt a. M.: Suhrkamp, 134-149.

Boltanski, Luc (2010): Soziologie und Sozialkritik. Frankfurt a. M.: Suhrkamp.

Bora, Alfons (2016): Responsive Rechtssoziologie. Zeitschrift für Rechtssoziologie 36, 261-272.

Bröckling, Ulrich (2013): Der Kopf der Leidenschaft. Soziologie und Kritik. Leviathan 41, 309-323.

Dahms, Hans-Joachim (2013): Bemerkungen zur Geschichte des Werturteilsstreits, in: Martin Carrier/Gerhard Schurz (Hrsg.), Werte in den Wissenschaften. Neue Ansätze zum Werturteilsstreit. Berlin: Suhrkamp, 74-107.

Dörre, Klaus/Lessenich, Stephan/Rosa, Hartmut (2009): Soziologie - Kapitalismus - Kritik. Eine Debatte. Frankfurt a. M.: Suhrkamp.

Fischer-Lescano, Andreas (2013): Systemtheorie als kritische Gesellschaftstheorie, in: Marc Amstutz/Andreas Fischer-Lescano (Hrsg.), Kritische Systemtheorie. Zur Evolution einer normativen Theorie. Bielefeld: transcript, 13-37. 
Füllsack, Manfred (1997): Dialektik der Post-Aufklärung. Zur Situation der kritischen Gesellschaftstheorie 50 Jahre nach Erscheinen der ,Dialektik der Aufklärung‘. Soziale Welt 48, 3, 313-328.

Gebhard, Gunther/Meißner, Stefan/Schröter, Steffen (2006): Kritik der Gesellschaft? Anschlüsse bei Luhmann und Foucault. Zeitschrift für Soziologie 35, 269-285.

Greve, Jens (2015): Gesellschaftskritik und die Krise der kritischen Theorie, in: Stephan Lessenich (Hrsg.), Routinen der Krise - Krise der Routinen. Verhandlungen des 37. Kongresses der Deutschen Gesellschaft für Soziologie in Trier 2014.

Honneth, Axel (2007): Rekonstruktive Gesellschaftskritik unter genealogischem Vorbehalt. Zur Idee der ,Kritik‘ in der Frankfurter Schule, in: ders., Pathologien der Vernunft. Geschichte und Gegenwart der kritischen Theorie. Frankfurt a. M.: Suhrkamp, 57-69.

Honneth, Axel (2014): Die Krankheiten der Gesellschaft. Annährungen an einen nahezu unmöglichen Begriff. WestEnd: Neue Zeitschrift für Sozialforschung 11, 1, 45-60.

Horster, Detlef (2008): Nachwort, in: Niklas Luhmann, Die Moral der Gesellschaft, hrsgg. von Detlef Horster. Frankfurt a. M.: Suhrkamp, 375-392.

Hume, David (1739): A Treatise of Human Nature. Oxford: Oxford University Press (Neudruck 1978).

Jaeggi, Rahel (2009): Was ist Ideologiekritik?, in: Rahel Jaeggi/Tilo Wesche (Hrsg.), Was ist Kritik? Frankfurt a. M.: Suhrkamp, 266-295.

Jaeggi, Rahel (2014): Kritik von Lebensformen. Berlin: Suhrkamp.

Jaeggi, Rahel/Wesche, Tilo (Hrsg.) (2009): Was ist Kritik? Frankfurt a. M.: Suhrkamp.

Japp, Klaus Peter (1991): Systemtheorie und Kritik, in: Harald Kerber (Hrsg.), Soziologie. Arbeitsfelder, Theorien, Ausbildung - ein Grundkurs. Reinbek bei Hamburg: Rowohlt, 579-594.

Johnson, Harry M. (1961): Sociology. A Systemic Introduction. London: Routledge.

Kieserling, André (2004a): Die Selbstbeschreibung der Soziologie, in: ders., Selbstbeschreibung und Fremdbeschreibung. Beiträge zur Soziologie soziologischen Wissens.

Frankfurt a. M.: Suhrkamp, 16-45.

Kieserling, André (2004b): Die Soziologie der Selbstbeschreibung. Über die Reflexionstheorien der Funktionssysteme und ihre Rezeption in der soziologischen Theorie, in: ders., Selbstbeschreibung und Fremdbeschreibung. Beiträge zur Soziologie soziologischen Wissens. Frankfurt a. M.: Suhrkamp, 46-108.

Kieserling, André (2012): Zweckbegriff und Systemrationalität. Über die Funktion von Zwecken in sozialen Systemen, in: Oliver Jahraus/Armin Nassehi et al. (Hrsg.), Luhmann-Handbuch. Leben - Werk - Wirkung. Stuttgart: Metzler, 135-140.

Kieserling, André (2014): Systemreverenzen. Wie wertet die Theorie sozialer Systeme? Zu Niklas Luhmann: ,Die Praxis der Theorie', Soziale Welt 20/2 (1969), in: Norman Braun et al (Hrsg.), Begriffe - Positionen - Debatten. Eine Relektüre von 65 Jahren Soziale Welt (Soziale Welt, Sonderband 21). Baden-Baden: Nomos, 89-98.

Kieserling, André (2015): Soziologische Ausgangspunkte für systemimmanente Kritik, in: Albert Scherr (Hrsg.), Systemtheorie und Differenzierungstheorie als Kritik. Weinheim, Basel: Beltz-Juventa, 140-153.

Kneer, Georg (1996): Rationalisierung, Disziplinierung und Differenzierung. Zum Zusammenhang von Sozialtheorie und Zeitdiagnose bei Jürgen Habermas, Michel Foucault und Niklas Luhmann. Opladen: Westdeutscher Verlag.

Lepsius, Mario Rainer (1964): Kritik als Beruf. Zur Soziologie der Intellektuellen. Kölner Zeitschrift für Soziologie und Sozialpsychologie 16, 75-91. 
Lessenich, Stephan (2014): Soziologie - Krise - Kritik. Zu einer kritischen Soziologie der Kritik. Soziologie 43, 1, 7-24.

Luhmann, Niklas (o.J.): Formale Struktur und richtiges Handeln. Bielefeld: Ms. (undatiertes Manuskript aus dem Nachlass; vermutlich Anfang 1960er Jahre verfasst).

Luhmann, Niklas (1962): Funktion und Kausalität, in: ders. (1970), Soziologische Aufklärung 1. Aufsätze zur Theorie sozialer Systeme. Opladen: Westdeutscher Verlag, 9-30.

Luhmann, Niklas (1964a): Funktionale Methode und Systemtheorie, in: ders. (1970), Soziologische Aufklärung 1: Aufsätze zur Theorie sozialer Systeme. Opladen: Westdeutscher Verlag, 31-53.

Luhmann, Niklas (1964b): Funktionen und Folgen formaler Organisation. Berlin: Duncker \& Humblot.

Luhmann, Niklas (1965a): Die Grenzen einer betriebswirtschaftlichen Verwaltungslehre. Verwaltungsarchiv 56, 303-313.

Luhmann, Niklas (1965b): Grundrechte als Institution. Ein Beitrag zur politischen Soziologie. Berlin: Duncker \& Humblot.

Luhmann, Niklas (1966a): Theorie der Verwaltungswissenschaft. Bestandsaufnahme und Entwurf. Köln: Grote.

Luhmann, Niklas (1966b): Recht und Automation in der öffentlichen Verwaltung. Eine verwaltungswissenschaftliche Untersuchung. Berlin: Duncker \& Humblot.

Luhmann, Niklas (1967a): Soziologie als Theorie sozialer Systeme, in: ders. (1970), Soziologische Aufklärung 1: Aufsätze zur Theorie sozialer Systeme. Opladen: Westdeutscher Verlag, 113-136.

Luhmann, Niklas (1967b): Soziologische Aufklärung, in: ders. (1970), Soziologische Aufklärung 1: Aufsätze zur Theorie sozialer Systeme. Opladen: Westdeutscher Verlag, 66-91.

Luhmann, Niklas (1968a): Status Quo als Argument, in: ders. (1992), Universität als Milieu, hrsgg. von André Kieserling. Bielefeld: Haux, 16-29.

Luhmann, Niklas (1968b): Zweckbegriff und Systemrationalität. Über die Funktion von Zwecken in sozialen Systemen. Frankfurt a. M.: Suhrkamp (Neudruck 1973).

Luhmann, Niklas (1969): Die Praxis der Theorie, in: ders. (1970), Soziologische Aufklärung 1: Aufsätze zur Theorie sozialer Systeme. Opladen: Westdeutscher Verlag, 253-267.

Luhmann, Niklas (1970a): Gesellschaft, in: ders., Soziologische Aufklärung 1: Aufsätze zur Theorie sozialer Systeme. Opladen: Westdeutscher Verlag, 137-153.

Luhmann, Niklas (1970b): Öffentliche Meinung, in: ders. (1971), Politische Planung. Aufsätze zur Soziologie von Politik und Verwaltung. Opladen: Westdeutscher Verlag, 9-34.

Luhmann, Niklas (1971): Reform des öffentlichen Dienstes. Zum Problem ihrer Probleme, in: ders., Politische Planung. Aufsätze zur Soziologie von Politik und Verwaltung. Opladen: Westdeutscher Verlag, 203-256.

Luhmann, Niklas (1974): ,Nomologische Hypothesen', funktionale Äquivalenz, Limitationalität. Zum wissenschaftstheoretischen Verständnis des Funktionalismus. Soziale Systeme 16 (2010), 1, 3-27.

Luhmann, Niklas (1977): Probleme eines Parteiprogramms, in: Horst Baier (Hrsg.), Freiheit und Sachzwang. Beiträge zu Ehren Helmut Schelskys. Opladen: Westdeutscher Verlag, 167-181.

Luhmann, Niklas (1978): Soziologie der Moral, in: Niklas Luhmann/Stephan H. Pfürtner (Hrsg.), Theorietechnik und Moral. Frankfurt a. M.: Suhrkamp, 8-116. 
Luhmann, Niklas (o.J. [1982]): Rationalität in der modernen Gesellschaft, in: ders. (2008), Ideenevolution. Beiträge zur Wissenssoziologie, hrsgg. von André Kieserling, Frankfurt a. M.: Suhrkamp, 186-233 (undatiertes Manuskript; vermutlich 1982 verfasst).

Luhmann, Niklas (1983): Interdisziplinäre Theoriebildung in den Sozialwissenschaften, in: ders. (1992), Universität als Milieu, hrsgg. von André Kieserling. Bielefeld: Haux, 62-68.

Luhmann, Niklas (1984): Soziale Systeme. Grundriß einer allgemeinen Theorie. Frankfurt a. M.: Suhrkamp.

Luhmann, Niklas (1986): Ökologische Kommunikation. Kann die moderne Gesellschaft sich auf ökologische Gefährdungen einstellen? Wiesbaden: VS (Neudruck 2008).

Luhmann, Niklas (1988): Die Wirtschaft der Gesellschaft. Frankfurt a. M.: Suhrkamp.

Luhmann, Niklas (1990): Die Wissenschaft der Gesellschaft. Frankfurt a. M.: Suhrkamp.

Luhmann, Niklas (1992): Die Selbstbeschreibung der Gesellschaft und die Soziologie, in: ders., Universität als Milieu, hrsgg. von André Kieserling. Bielefeld: Haux, 137-146.

Luhmann, Niklas (1993a): Das Recht der Gesellschaft. Frankfurt a. M.: Suhrkamp.

Luhmann, Niklas (1993b): Die Ehrlichkeit der Politiker und die höhere Amoralität der Politik, in: ders. (2008), Die Moral der Gesellschaft, hrsgg. von Detlef Horster. Frankfurt a. M.: Suhrkamp, 163-174.

Luhmann, Niklas (1993c): Gibt es in unserer Gesellschaft noch unverzichtbare Normen?, in: ders. (2008), Die Moral der Gesellschaft, hrsgg. von Detlef Horster. Frankfurt a. M.: Suhrkamp, 228-252.

Luhmann, Niklas (1993d): Quod omnes tangit ... Anmerkungen zur Rechtstheorie von Jürgen Habermas. Rechtshistorisches Journal 12, 36-56.

Luhmann, Niklas (1993e): „Was ist der Fall?“ und „Was steckt dahinter?“. Die zwei Soziologien und die Gesellschaftstheorie. Zeitschrift für Soziologie 22, 4, 245-260.

Luhmann, Niklas (1996): Die neuzeitlichen Wissenschaften und die Phänomenologie. Vortrag im Wiener Rathaus am 25. Mai 1995. Wien: Picus Verlag.

Luhmann, Niklas (1997): Die Gesellschaft der Gesellschaft. 2 Bd. Frankfurt a. M.: Suhrkamp.

Mannheim, Karl (1927): Das konservative Denken. Soziologische Beiträge zum Werden des politisch-historischen Denkens in Deutschland, in: ders. (1970), Wissenssoziologie. Auswahl aus dem Werk. Herausgegeben von Kurt H. Wolff. Neuwied, Berlin: Luchterhand, 408-508.

Merton, Robert King (1957): Soziologische Theorie und soziale Struktur. Berlin: de Gruyter (Neudruck 1995).

Mills, Charles Wright (1963): Soziologische Phantasie. Herausgegeben von Stephan Lessenich. Wiesbaden: VS (Neudruck 2016).

Möller, Kolja/Siri, Jasmin (Hrsg.) (2016): Systemtheorie und Gesellschaftskritik. Perspektiven der Kritischen Systemtheorie. Bielefeld: transcript.

Müller-Doohm, Stefan (2001): Soziologie als Aufklärungswissenschaft, in: Schweizerische Zeitschrift für Soziologie 27, 3, 379-391.

Musil, Robert (1930): Der Mann ohne Eigenschaften. Roman. Reinbek bei Hamburg: Rowohlt (Neudruck 1987).

Myrdal, Gunnar (1944): An American dilemma. The Negro problem and modern democracy. New York: Harper \& Row.

Myrdal, Gunnar (1971): Noch einmal: Ein amerikanisches Dilemma. Die Rassenkrise in den Vereinigten Staaten in historischer Perspektive, in: ders., Aufsätze und Reden. Frankfurt a. M.: Suhrkamp, 106-155. 
Opitz, Sven (2013): Was ist Kritik? Was ist Aufklärung? Zum Spiel der Möglichkeiten bei Niklas Luhmann und Michel Foucault, in: Marc Amstutz/Andreas Fischer-Lescano (Hrsg.), Kritische Systemtheorie. Zur Evolution einer normativen Theorie. Bielefeld: transcript, 39-62.

Pahl, Hanno (2009): Soziologische Aufklärung zwischen Kritik, Affirmation und Normativität. Implikationen der Theorie sozialer Systeme für das Projekt einer Fortschreibung der kritischen Theorie, in: Devi Dumbadze (Hrsg.), Erkenntnis und Kritik. Zeitgenössische Positionen. Bielefeld: transcript, 241-261.

Peters, Bernhard (1991): Rationalität, Recht und Gesellschaft. Frankfurt a. M.: Suhrkamp. Pollack, Detlef (1991): Bestandserhaltung oder Kritik oder: weder Bestandserhaltung noch Kritik. Die Intention der Systemtheorie Niklas Luhmanns. Deutsche Zeitschrift für Philosophie, 39, 1, 95-99.

Richter, Dirk (2011): Normativität in der Systemtheorie, in: Johannes Ahrens/Raphael Beer et al. (Hrsg.), Normativität. Über die Hintergründe sozialwissenschaftlicher Theoriebildung. Wiesbaden: VS, 271-285.

Röttgers, Kurt (1982): Kritik, in: Otto Brunner/Werner Conze/Reinhart Koselleck (Hrsg.), Geschichtliche Grundbegriffe: historisches Lexikon zur politisch-sozialen Sprache in Deutschland. Bd. 3: H - Me. Stuttgart: Klett-Cotta, 651-675.

Scherr, Albert (2015) Keine 11. These mehr? Niklas Luhmann als kritischer Theoretiker der Gegenwartsgesellschaft, in: ders. (Hrsg.), Systemtheorie und Differenzierungstheorie als Kritik. Weinheim, Basel: Beltz-Juventa, 13-36.

Schmidt, Johannes F.K. (2013): Zum Verhältnis von Rechtstheorie und Rechtssoziologie bei Niklas Luhmann. Editorische Notiz, in: Niklas Luhmann: Kontingenz und Recht. Rechtstheorie im interdisziplinären Zusammenhang, hrsgg. von Johannes F.K. Schmidt. Berlin: Suhrkamp, 330-344.

Schroer, Markus (2004): Zwischen Engagement und Distanzierung. Zeitdiagnose und Kritik bei Pierre Bourdieu und Niklas Luhmann, in: Armin Nassehi (Hrsg.), Bourdieu und Luhmann. Frankfurt a. M.: Suhrkamp, 233-271.

Sloterdijk, Peter (2010): Luhmann, Anwalt des Teufels, in: Wolfram Burckhardt (Hrsg.), Luhmann-Lektüren. Berlin: Kulturverlag Kadmos, 91-158.

Stäheli, Urs (2012): Luhmanns „Soziologische Aufklärung“, in: Dietmar J. Wetzel (Hrsg.), Perspektiven der Aufklärung. Zwischen Mythos und Realität. München, Paderborn: Fink, $35-42$.

Stahl, Titus (2013): Immanente Kritik. Elemente einer Theorie sozialer Praktiken. Frankfurt: Campus.

Stahl, Titus (2014): Die normativen Grundlagen immanenter Kritik. Sozialontologische Überlegungen zum Potenzial sozialer Praktiken, in: José M. Romero (Hrsg.), Immanente Kritik heute. Grundlagen und Aktualität eines sozialphilosophischen Begriffs. Bielefeld: transcript, 31-57.

Tacke, Veronika/Kette, Sven (2015): Systemtheorie, Organisation und Kritik, in: Albert Scherr (Hrsg.), Systemtheorie und Differenzierungstheorie als Kritik. Weinheim, Basel: BeltzJuventa, 232-265.

Vobruba, Georg (2013): Soziologie und Kritik. Moderne Sozialwissenschaft und Kritik der Gesellschaft. Soziologie 42, 147-168.

Wagner, Elke (2005): Gesellschaftskritik und soziologische Aufklärung. Konvergenzen und Divergenzen zwischen Adorno und Luhmann. Berliner Journal für Soziologie 35, 37-54. 
Wagner, Elke (2012): Theorie ohne Kritik?, in: Oliver Jahraus/Armin Nassehi et al (Hrsg.), Luhmann-Handbuch. Leben - Werk - Wirkung. Stuttgart: Metzler, 428-431.

Wagner, Elke (2013): Systemtheorie und Frankfurter Schule, in: Marc Amstutz/Andreas

Fischer-Lescano (Hrsg.), Kritische Systemtheorie. Zur Evolution einer normativen Theorie. Bielefeld: transcript, 63-80.

Weber, Max (1904): Die „Objektivität“ sozialwissenschaftlicher und sozialpolitischer Erkenntnis, in: ders. (1985), Gesammelte Aufsätze zur Wissenschaftslehre: Mohr Siebeck, 146-214.

Weber, Max (1917): Der Sinn der ,Wertfreiheit' der soziologischen und ökonomischen Wissenschaften, in: Martin Carrier/Gerhard Schurz (Hrsg.) (2013), Werte in den Wissenschaften. Neue Ansätze zum Werturteilsstreit. Berlin: Suhrkamp, 33-56.

Weber, Max (1919): Wissenschaft als Beruf. Stuttgart: Reclam (Neudruck 1995).

\section{Autoreninformationen}

\section{Martin Weißmann}

Fakultät für Soziologie, Universität Bielefeld, Postfach 100 131, D-33501 Bielefeld, email: martin.weissmann@uni-bielefeld.de

Martin Weißmann ist wissenschaftlicher Mitarbeiter am Arbeitsbereich Theoretische Soziologie der Fakultät für Soziologie der Universität Bielefeld. Seine Arbeitsfelder umfassen vor allem Fragestellungen der soziologischen Theorie und der Organisationssoziologie. Aktuelle Publikation: Organisierte Entmenschlichung. Zur Produktion, Funktion und Ersetzbarkeit sozialer und psychischer Dehumanisierung in Genoziden, in: Alexander Gruber/Stefan Kühl (Hrsg.), Soziologische Analysen des Holocaust. Jenseits der Debatte über „ganz normale Männer“ und „ganz normale Deutsche“. Wiesbaden: VS 2015, 79-128. 